Supporting Information:

\title{
Stereoselective Carbon-Carbon Bond Formation via the Mitsunobu Displacement of Chiral Secondary Benzylic Alcohols.
}

\author{
Michael C. Hillier,* Jean-Nicolas Desrosiers, Jean-François Marcoux, Edward J. J. Grabowski \\ Department of Process Research, Merck \& Co.,Inc., P.O. Box 2000, Rahway, NJ 07065.
}

\section{Experimentals-}

\section{General:}

Unless otherwise stated, solvents and reagents were reagent grade and used without further purification. Melting points were determined on a Büchi B-545 melting point apparatus and are uncorrected. Infrared (IR) spectra were recorded on a Nicolet Magna-IR 560 spectrometer using PTFE IR cards, and are reported in wavenumbers $\left(\mathrm{cm}^{-1}\right) .{ }^{1} \mathrm{H}(400 \mathrm{MHz})$ and ${ }^{13} \mathrm{C}(100$ MHz) NMR spectra were obtained using a Bruker $400 \mathrm{MHz}$ instrument, and chemical shifts are reported in parts per million (ppm). Coupling constants are reported in hertz $(\mathrm{Hz})$. Spectral splitting patterns are designated as: s, singlet; br, broad; d, doublet; t, triplet; q, quartet; m, multiplet; and comp, complex multiplet. Elemental analysis was performed by QTI technologies (Whitehouse, NJ) and hi-resolution mass spectra were obtained using an electrospray mass spectrometer.

The following chiral alcohols were purchased: $(R)$ - $\alpha$-methyl-2-napthalenemethanol (3, Aldrich), $(R)$-1-phenyl-1-ethanol (6a, Lancaster), $(R)$-1-phenyl-1-butanol (6d, Aldrich), $(R)$-2-methyl-1-phenyl-1-propanol (6e, Lancaster), 1-(S)-indanol (6f, Aldrich). The following chiral alcohols were prepared according to known procedures: $(R)-1-[4-($ methoxylphenyl)]-ethanol $(\mathbf{9 a}),{ }^{1}(R)$-1-[4-trifluoromethylphenyl)]-ethanol $(\mathbf{6 b}),(R)$-1-[4-(chlorophenyl)]-ethanol $(\mathbf{6 c}),{ }^{1}(R)$-1-tetrahydronapthol $(\mathbf{6 g}),{ }^{2}$ $(R)$ - $\alpha$-(3,4,5-trimethoxyphenyl)-1,3-benzodioxole-5-methanol $(\mathbf{9 b}),{ }^{3}$ 2-methyl-(S)-benzhydrol $(\mathbf{9 c}){ }^{3}$

\section{General Mitsunobu Procedure:}

To a solution of the alcohol $(1 \mathrm{mmol})$ and triethylmethanetricarboxylate $(2 \mathrm{mmol})$ in THF:toluene $(1: 1 \mathrm{or} 1: 4)$ or toluene (4 $\mathrm{mL})$ at $\mathrm{rt}$ was added $\mathrm{PMe}_{3}(2 \mathrm{mmol})$ and the resulting solution was cooled to $-78{ }^{\circ} \mathrm{C}$. DEAD $(2 \mathrm{mmol})$ was then added slowly at such a rate as to keep the internal temperature of the reaction below $-65^{\circ} \mathrm{C}$. The reaction was stirred for $0.5 \mathrm{~h}$ at $78{ }^{\circ} \mathrm{C}$ and warmed to $\mathrm{rt}$ for $1-3 \mathrm{~h}$, whereupon solvent was concentrated in vacuo. The resulting crude oil was taken up in MTBE $(10 \mathrm{~mL})$ and washed with $3.3 \mathrm{~N} \mathrm{NaOH}(2 \times 2 \mathrm{~mL}), 1 \mathrm{~N} \mathrm{HCl}(2 \mathrm{~mL})$, dried $\left(\mathrm{MgSO}_{4}\right)$, concentrated, and the crude product purified via column chromatography eluting with EtOAc/Hexanes.

Triethyl (2S)-2-(2-napthyl)propane-1,1,1-tricarboxylate (4). Obtained as an oil from 3 according to the general Mitsunobu procedure in 96\% ee; chiral SFC: Chirapak AD column, 4\% EtOH in $\mathrm{CO}_{2}$ for 4 min. then ramp to $40 \% \mathrm{EtOH} @$ $2 \%$ per min, $t_{\mathrm{R}}=5.1 \mathrm{~min} .(R), 6.3 \mathrm{~min} .(S) ;{ }^{1} \mathrm{H}$ NMR $\delta 7.87(\mathrm{~s}, 1 \mathrm{H}), 7.80-7.74(\mathrm{~m}, 3 \mathrm{H}), 7.57(\mathrm{dd}, J=1.7,8.6 \mathrm{~Hz}, 1 \mathrm{H})$, 7.46-7.41 (m, $2 \mathrm{H}), 7.24-4.11(\mathrm{~m}, 6 \mathrm{H}), 4.04(\mathrm{q}, J=7.2 \mathrm{~Hz}), 1.60(\mathrm{~d}, J=7.2 \mathrm{~Hz}, 3 \mathrm{H}), 1.18(\mathrm{t}, J=7.2 \mathrm{~Hz}, 9 \mathrm{H}) ;{ }^{13} \mathrm{C}$ NMR $\delta$ 166.4, 139.2, 133.2, 132.6, 128.3, 128.0, 127.8, 127.5, 127.3, 125.8, 125.7, 70.0, 61.9, 43.7, 19.0, 13.9; IR v 2982, 1743, 1231, 1154, 1096, $1042 \mathrm{~cm}^{-1}$; mass spectrum (ESI) calcd. for $\mathrm{C}_{22} \mathrm{H}_{26} \mathrm{O}_{6}+\mathrm{Li}: \mathrm{M}+\mathrm{Li}$ (theory), 392.1880; $\mathrm{M}+\mathrm{Li}$ (found), 392.1882 .

Triethyl (2S)-2-phenylpropane-1,1,1-tricarboxylate (7a). Obtained as an oil from 6a according to the general Mitsunobu procedure; ${ }^{1} \mathrm{H}$ NMR data matched that for the known racemic compound. ${ }^{4}{ }^{13} \mathrm{C}$ NMR $\delta 166.1,141.4,129.2,127.7,126.9$, 69.5, 61.6, 43.4 18.8, 13.7; IR $\vee 2982,2940,1743,1455,1204,1151,1102,1040,701 \mathrm{~cm}^{-1}$; mass spectrum (ESI) calcd. for $\mathrm{C}_{18} \mathrm{H}_{24} \mathrm{O}_{6}+\mathrm{Li}: \mathrm{M}+\mathrm{Li}$ (theory), 342.1724; $\mathrm{M}+\mathrm{Li}$ (found), 342.1725 .

Triethyl (2S)-2-[4-(trifluoromethyl)propane-1,1,1-tricarboxylate (7b). Obtained as an oil from $6 \mathbf{b}$ according to the general Mitsunobu procedure; ${ }^{1} \mathrm{H}$ NMR $\delta 7.57-7.50(\mathrm{comp}, 4 \mathrm{H}), 4.24-4.11(\mathrm{~m}, 6 \mathrm{H}), 3.90(\mathrm{q}, J=7.2 \mathrm{~Hz}, 1 \mathrm{H}), 1.51(\mathrm{~d}, J=$ $7.2 \mathrm{~Hz}, 3 \mathrm{H}), 1.19(\mathrm{dt}, J=0.5,7.2 \mathrm{~Hz}, 9 \mathrm{H}) ;{ }^{13} \mathrm{C}$ NMR $\delta 166.1,145.8,129.7,129.3\left(\mathrm{q}, J_{\mathrm{CF}}=32.5 \mathrm{~Hz}\right), 124.7\left(\mathrm{q}, J_{C F}=3.8 \mathrm{~Hz}\right)$ $124.2\left(\mathrm{q}, J_{\mathrm{CF}}=271.9 \mathrm{~Hz}\right) 69.4,62.0,43.2,18.7,13.7$; IR $\vee 2983,1741,1326,1205,1152,1122,847 \mathrm{~cm}^{-1}$; mass spectrum (ESI) calcd. for $\mathrm{C}_{19} \mathrm{H}_{23} \mathrm{~F}_{3} \mathrm{O}_{6}+\mathrm{Li}$ : $\mathrm{M}+\mathrm{Li}$ (theory), 410.1598; $\mathrm{M}+\mathrm{Li}$ (found), 410.1601 .

\footnotetext{
${ }^{1}$ Fujii, A.; Hashiguchi, S.; Uematsu, N.; Ikariya, T.; Noyori, R. J. Am. Chem. Soc. 1996, 118, 2521-2522.

${ }^{2}$ Quallich, G. J.; Woodall, T. M. Tetrahedron Lett. 1993, 34, 4145-4148.

${ }^{3}$ Corey, E. J.; Helal, C. J. Tetrahedron Lett. 1996, 37, 5675-5678.

${ }^{4}$ Cravotto, G.; Giovenzana, G. B.; Sisti, M.; Palmisano, G. Tetrahedron 1996, 52, 13007-13016.
} 
Triethyl (2S)-2-(4-chlorophenyl)propane-1,1,1-tricarboxylate (7c). Obtained as an oil from $\mathbf{6 c}$ according to the general Mitsunobu procedure; ${ }^{1} \mathrm{H}$ NMR $\delta 7.36(\mathrm{dd}, J=2.0,2.6 \mathrm{~Hz}, 1 \mathrm{H}), 7.35(\mathrm{dd}, J=2.0,2.6 \mathrm{~Hz}, 1 \mathrm{H}), 7.23(\mathrm{dd}, J=2.0,2.6 \mathrm{~Hz}, 1$ H), $7.20(\mathrm{dd}, J=2.0,2.6 \mathrm{~Hz}, 1 \mathrm{H}), 4.21-4.10(\mathrm{~m}, 6 \mathrm{H}), 3.82(\mathrm{q}, J=7.2 \mathrm{~Hz}, 1 \mathrm{H}), 1.47(\mathrm{~d}, J=7.2 \mathrm{~Hz}, 3 \mathrm{H}), 1.20(\mathrm{t}, J=7.2$ $\mathrm{Hz}) ;{ }^{13} \mathrm{C}$ NMR $\delta 166.1,140.1,132.8,130.8,127.9,69.5,61.9,42.8,18.8,13.8,13.7 ;$ IR $\vee 2982,1742,1493,1206,1151$, 1093, $1040 \mathrm{~cm}^{-1}$; mass spectrum (ESI) calcd. for $\mathrm{C}_{18} \mathrm{H}_{23} \mathrm{ClO}_{6}+\mathrm{Li}$ : $\mathrm{M}+\mathrm{Li}$ (theory), 376.1335; $\mathrm{M}+\mathrm{Li}$ (found), 376.1330 .

Triethyl (2S)-2-phenylpentane-1,1,1-tricarboxylate (7d). Obtained as an oil from 6d according to the general Mitsunobu procedure; ${ }^{1} \mathrm{H}$ NMR $\delta$ 7.39-7.17 (comp, $\left.5 \mathrm{H}\right), 4.22-4.09(\mathrm{~m}, 6 \mathrm{H}), 3.60(\mathrm{dd}, J=4.8,8.9 \mathrm{~Hz}, 1 \mathrm{H}), 1.91-1.81(\mathrm{~m}, 2 \mathrm{H}), 1.19(\mathrm{t}$, $J=7.2 \mathrm{~Hz}, 3 \mathrm{H}), 1.14-1.06(\mathrm{~m}, 2 \mathrm{H}), 0.84(\mathrm{t}, J=7.3 \mathrm{~Hz}, 3 \mathrm{H}) ;{ }^{13} \mathrm{C}$ NMR $\delta 166.4,129.4,130.1,128.4,127.7,69.8,61.7,49.1$, 34.6, 20.9, 13.8; IR $\vee 2961,2936,1872,1743,1455,12131056 \mathrm{~cm}^{-1}$; mass spectrum (ESI) calcd. for $\mathrm{C}_{20} \mathrm{H}_{28} \mathrm{O}_{6}+\mathrm{Li}: \mathrm{M}+\mathrm{Li}$ (theory), 370.2037; M+Li (found), 370.2035.

Triethyl (1R)-2,3-dihydro-1H-inden-1-ylmethanetricarboxylate (7f). Obtained as an oil from $\mathbf{6 f}$ according to the general Mitsunobu procedure in $87 \%$ ee; chiral SFC: Chiralcel OD-H column, $4 \% \mathrm{EtOH}$ in $\mathrm{CO}_{2}$ for 4 min. then ramp to $40 \% \mathrm{EtOH}$ @ $2 \%$ per min, $t_{\mathrm{R}}=3.8 \mathrm{~min}$. $(R), 4.1 \mathrm{~min} .(S)$; ${ }^{1} \mathrm{H}$ NMR $\delta$ 7.40-7.32 $(\mathrm{m}, 1 \mathrm{H}), 7.20-7.05(\mathrm{~m}, 3 \mathrm{H}), 4.30-4.24(\mathrm{~m}, 1 \mathrm{H}), 4.23-$ $4.13(\mathrm{~m}, 6 \mathrm{H}), 2.95$ (dddd, $J=4.5,9.2,15.8,15.8 \mathrm{~Hz}, 1 \mathrm{H}), 2.86$ (ddd, $J=8.3,15.8,15.8 \mathrm{~Hz}, 1 \mathrm{H}), 2.41$ (dddd, $J=4.5,8.7$, 8.7, $13.2 \mathrm{~Hz}, 1 \mathrm{H}), 2.26$ (dddd, $J=7.9,9.4,15.8,15.8 \mathrm{~Hz}, 1 \mathrm{H}), 1.18(\mathrm{t}, J=7.2 \mathrm{~Hz}, 9 \mathrm{H}) ;{ }^{13} \mathrm{C}$ NMR $\delta 166.6,144.8,141.5$, 127.1, 126.0, 125.6, 124.2, 70.0, 61.9, 48.1, 31.0, 28.9, 13.7; IR $\vee 2982,1741,1458,1260,1208,1152,1061 \mathrm{~cm}^{-1}$; mass spectrum (ESI) calcd. for $\mathrm{C}_{19} \mathrm{H}_{24} \mathrm{O}_{6}+\mathrm{Li}$ : $\mathrm{M}+\mathrm{Li}$ (theory), 354.1724; $\mathrm{M}+\mathrm{Li}$ (found), 354.1729.

Triethyl (1S)-1,2,3,4-tetrahydronapthalene-1-ylmethanetricarboxylate (7g). Obtained as an oil from $\mathbf{6 g}$ according to the general Mitsunobu procedure; ${ }^{1} \mathrm{H}$ NMR $\delta$ 7.41-7.40 (m, $\left.1 \mathrm{H}\right), 7.10-7.00(\mathrm{~m}, 3 \mathrm{H}), 4.14(\mathrm{q}, J=7.2 \mathrm{~Hz}, 6 \mathrm{H}), 4.20-4.10(\mathrm{~m}, 1$ $\mathrm{H}), 2.83-2.68(\mathrm{~m}, 2 \mathrm{H}), 2.11-1.95(\mathrm{~m}, 3 \mathrm{H}), 1.71-1.60(\mathrm{~m}, 1 \mathrm{H}), 1.17(\mathrm{t}, J=7.2 \mathrm{~Hz}, 9 \mathrm{H}) ;{ }^{13} \mathrm{C}$ NMR $\delta 166.7,139.0,135.7$, 128.8, 128.7, 126.1, 125.3, 71.2, 61.8, 40.9, 30.1, 27.0, 22.0, 13.6; IR $\vee 2937,1738,1447,1206,1150,1057 \mathrm{~cm}^{-1}$; mass spectrum (ESI) calcd. for $\mathrm{C}_{20} \mathrm{H}_{26} \mathrm{O}_{6}+\mathrm{Li}$ : M+Li (theory), 368.1880; $\mathrm{M}+\mathrm{Li}$ (found), 368.1883.

Triethyl (2S)-2-(4-methoxyphenyl)propane-1,1,1-tricarboxylate (10a). Obtained as an oil from 9a according to the general Mitsunobu procedure; ${ }^{1} \mathrm{H}$ NMR $\delta$ 7.35-7.31 (comp, $2 \mathrm{H}$ ), 6.81-6.77 (comp, $\left.2 \mathrm{H}\right), 4.16$ (m, $\left.6 \mathrm{H}\right), 3.81$ (q, J = 7.2 Hz, 1 $\mathrm{H}), 3.78(\mathrm{~s}, 3 \mathrm{H}), 1.47(\mathrm{~d}, J=7.2 \mathrm{~Hz}, 3 \mathrm{H}), 1.20(\mathrm{t}, J=7.2 \mathrm{~Hz}, 9 \mathrm{H}) ;{ }^{13} \mathrm{C}$ NMR $\delta 166.4,158.6,133.5,130.5,113.2,69.9,61.7$, 55.2, 42.8, 18.9, 13.9; IR $\vee 2982,2940,1744,1611,1514,1464,1367,1183,1095,1040 \mathrm{~cm}^{-1}$; mass spectrum (ESI) calcd. for $\mathrm{C}_{19} \mathrm{H}_{26} \mathrm{O}_{7}+\mathrm{Li}: \mathrm{M}+\mathrm{Li}$ (theory), 372.1830; $\mathrm{M}+\mathrm{Li}$ (found), 371.1833 .

Triethyl 2-(1,3-benzodioxol-5-yl)-2-(3,4,5-trimethoxyphenyl)ethane-1,1,1-tricarboxylate (10b). Obtained as a solid from 9b according to the general Mitsunobu procedure: m.p. $=90.1-90.2{ }^{\circ} \mathrm{C} ;{ }^{1} \mathrm{H}$ NMR 7.15-7.07 (m, $\left.2 \mathrm{H}\right), 6.90(\mathrm{~s}, 2 \mathrm{H})$, $6.70(\mathrm{~d}, J=8.0 \mathrm{~Hz}, 1 \mathrm{H}), 5.90(\mathrm{~s}, 2 \mathrm{H}), 5.20(\mathrm{~s}, 1 \mathrm{H}), 4.06-3.95(\mathrm{~m}, 6 \mathrm{H}), 3.84(\mathrm{~s}, 6 \mathrm{H}), 3.80(\mathrm{~s}, 3 \mathrm{H}), 1.02(\mathrm{t}, J=7.2 \mathrm{~Hz}, 9$ $\mathrm{H}) ;{ }^{13} \mathrm{C}$ NMR $\delta 166.2,152.7,147.2,146.4,136.5,135.9,133.5,123.3,110.5,107.7,106.8,100.9,70.5,60.8,56.0,53.3$, 13.5; IR $\vee 2981,1744,1589,1488,1207,1151,1128 \mathrm{~cm}^{-1}$; mass spectrum (ESI) calcd. for $\mathrm{C}_{27} \mathrm{H}_{32} \mathrm{O}_{11}+\mathrm{Li}$ : $\mathrm{M}+\mathrm{Li}$ (theory), 538.2096; M+Li (found), 538.2089.

Triethyl (2R)-2-(2-methylphenyl)-2-phenylethane-1,1,1-tricarboxylate (10c). Obtained as an oil from 9c according to the general Mitsunobu procedure; ${ }^{1} \mathrm{H}$ NMR $\left(\mathrm{CDCl}_{3}\right) \delta 8.23(\mathrm{~d}, J=7.8 \mathrm{~Hz}), 7.49(\mathrm{ddd}, J=1.4,1.4,7.3 \mathrm{~Hz}, 2 \mathrm{H}), 7.39-7.06$ (comp, $6 \mathrm{H}) 5.64(\mathrm{~s}, 1 \mathrm{H}), 4.04-3.86(\mathrm{comp}, 6 \mathrm{H}), 2.29(\mathrm{~s}, 3 \mathrm{H}), 0.95(\mathrm{t}, J=7.1 \mathrm{~Hz}, 9 \mathrm{H}) ;{ }^{13} \mathrm{C} \mathrm{NMR}\left(\mathrm{CDCl}_{3}\right) \delta 166.1,139.2,138.2$, 136.2, 130.6, 130.3, 128.1, 127.6, 126.7, 126.6, 126.0, 70.2, 61.7, 48.5, 20.0, 13.3; IR v 3061, 2981, 2361, 2337, 1779, 1635, $1540 \mathrm{~cm}^{-1}$; CHN calcd. for $\mathrm{C}_{24} \mathrm{H}_{28} \mathrm{O}_{6}$ [412.19]: C, 69.88; H, 6.84. Found: C, 69.81; H, 6.93.

General saponification and decarboxylation procedure: A solution of the triester adduct ( $1 \mathrm{mmol})$ in $\mathrm{MeOH}(3 \mathrm{~mL})$ was treated with a solution $3.3 \mathrm{~N} \mathrm{NaOH}(1.8 \mathrm{~mL}, 6 \mathrm{mmol})$ in water, the resulting mixture was heated to reflux, and the reaction was monitored by HPLC. When no starting material remained (6-18 hrs), heating was removed and the reaction was allowed to reach rt. Volatiles were removed in vacuo and the crude tris-acid was taken up in $\mathrm{AcOH}(6 \mathrm{~mL})$ and heated to reflux. The reaction was monitored by HPLC analysis, and when no starting material remained (6-18 hrs), solvent was concentrated in vacuo. The resultant crude acid was dissolved in water $(5 \mathrm{~mL})$, extracted with EtOAc $(3 \times 2 \mathrm{~mL})$, and the combined extracts were dried $\left(\mathrm{MgSO}_{4}\right)$ and concentrated in vacuo to give product. All acids were $>95 \%$ pure as judged by ${ }^{1} \mathrm{H}$ NMR analysis, and the enantiomeric purity of product was determined via chiral GC, SFC, or HPLC as indicated, compared to racemic standards.

(3R)-3-(2-Napthyl)butanoic acid (5). ${ }^{5}$ Obtained from 4 as an oil according to the general saponification and decarboxylation procedure; chiral SFC: Chiralcel OJ column, $4 \% \mathrm{MeOH}$ in $\mathrm{CO}_{2}$ for 4 min. then ramp to $40 \% \mathrm{MeOH}$ at $2 \%$ per min., $1.5 \mathrm{~mL} / \mathrm{min} ., 35^{\circ} \mathrm{C}, 25 \mathrm{~min}$. run time, $t_{\mathrm{R}}=12.1 \mathrm{~min}(R), 12.9 \mathrm{~min}(S)$.

\footnotetext{
${ }^{5}$ Lee, T.; Jones, J. B. J. Am. Chem. Soc. 1997, 119, 10260-10268.
} 
(3R)-3-Phenylbutanoic acid (8a). ${ }^{6}$ Obtained from $7 \mathbf{a}$ as a solid according to the general saponification and decarboxylation procedure; chiral SFC: Chiralcel OJ column, $4 \% \mathrm{EtOH}$ in $\mathrm{CO}_{2}$ for 4 min. then ramp to $40 \% \mathrm{EtOH}$ at $2 \%$ per min, 1.5 $\mathrm{mL} / \mathrm{min} ., 35^{\circ} \mathrm{C}, 25 \mathrm{~min}$. run time, $t_{\mathrm{R}}=6.3 \mathrm{~min} .(R), 7.0 \min (S)$.

(3R)-3-[4-(Trifluoromethyl)phenyl]butanoic acid (8b). Obtained from $\mathbf{7 b}$ as a solid according to the general saponification and decarboxylation procedure: m.p. $=117.6-117.7^{\circ} \mathrm{C}$; chiral GC: CyclosilC column (J\&W Scientific), 50$140{ }^{\circ} \mathrm{C}$ at $7{ }^{\circ} \mathrm{C} / \mathrm{min}$., hold at $140{ }^{\circ} \mathrm{C}$ for $60 \mathrm{~min} ., t_{\mathrm{R}}=49.5 \mathrm{~min} .(R), 50.6 \mathrm{~min} .(S) ;{ }^{1} \mathrm{H}$ NMR $\delta 12.1(\mathrm{brs}, 1 \mathrm{H}), 7.60(\mathrm{~d}, J=8.0$ $\mathrm{Hz}, 2 \mathrm{H}), 7.46(\mathrm{~d}, J=8.0 \mathrm{~Hz}, 2 \mathrm{H}), 3.25(\mathrm{dq}, J=7.0,7.5 \mathrm{~Hz}, 1 \mathrm{H}), 2.56(\mathrm{~d}, J=7.5 \mathrm{~Hz}, 2 \mathrm{H}), 1.22(\mathrm{~d}, J=7.0 \mathrm{~Hz}, 3 \mathrm{H}) ;{ }^{13} \mathrm{C}$ NMR $\delta 173.3,151.2,128.0,127.3\left(\mathrm{q}, J_{\mathrm{CF}}=31.9 \mathrm{~Hz}\right), 125.4\left(\mathrm{q}, J_{\mathrm{CF}}=3.6 \mathrm{~Hz}\right), 124.7(271.6 \mathrm{~Hz}), 41.9,36.1,21.9$; IR v; CHN calcd. for $\mathrm{C}_{11} \mathrm{H}_{11} \mathrm{~F}_{3} \mathrm{O}_{3}$ [248.07]: C, 56.90; H, 4.77. Found: C, 57.00; H, 4.72.

(3R)-3-(4-Chlorophenyl)butanoic acid (8c). ${ }^{7}$ Obtained from $7 \mathrm{c}$ as an oil according to the general saponification and decarboxylation procedure; chiral GC: Cyclosil C column (J\&W Scientific), 50-120 ${ }^{\circ} \mathrm{C}$ at $7{ }^{\circ} \mathrm{C} / \mathrm{min}$., hold at $120^{\circ} \mathrm{C}$ for 60 $\min ., t_{\mathrm{R}}=45.2 \min .(R), 45.8 \min (S)$.

(3R)-3-Phenylheptanoic acid (8d). ${ }^{8}$ Obtained from $7 \mathbf{d}$ as an oil according to the general saponification and decarboxylation procedure; chiral SFC: chiralcel OD column, $4 \% \mathrm{EtOH}$ in $\mathrm{CO}_{2}$ for 4 min. then ramp to $40 \% \mathrm{EtOH}$ at $2 \%$ per min, 1.5 $\mathrm{mL} / \mathrm{min} ., 35^{\circ} \mathrm{C}, 25 \mathrm{~min}$. run time, $t_{\mathrm{R}}=6.3 \mathrm{~min} .(R), 8.3 \min (S)$.

(1S)-2,3-Dihydro-1 $\mathrm{H}$-inden-1-ylacetic acid (8f). ${ }^{9}$ Obtained from $\mathbf{7 f}$ as an oil according to the general saponification and decarboxylation procedure; chiral SFC: chiralcel $\mathrm{AD}$ column, $4 \% \mathrm{EtOH}$ in $\mathrm{CO}_{2}$ for 4 min. then ramp to $40 \% \mathrm{EtOH}$ at $2 \%$ per min, $1.5 \mathrm{~mL} / \mathrm{min} ., 35^{\circ} \mathrm{C}, 25 \mathrm{~min}$. run time, $t_{\mathrm{R}}=9.7 \mathrm{~min} .(R), 10 \mathrm{~min}(S)$.

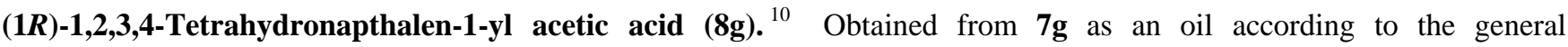
saponification and decarboxylation procedure; chiral SFC: chiralcel OD column, $4 \% \mathrm{EtOH}$ in $\mathrm{CO}_{2}$ for 4 min. then ramp to $40 \% \mathrm{EtOH}$ at $2 \%$ per min, $1.5 \mathrm{~mL} / \mathrm{min} ., 35^{\circ} \mathrm{C}, 25 \mathrm{~min}$. run time, $t_{\mathrm{R}}=6.3 \mathrm{~min} .(R), 8.3 \mathrm{~min}(S)$.

(3R)-3-(4-Methoxyphenyl)butanoic acid (11a). ${ }^{11}$ Obtained from 10a as an oil in $60 \%$ ee according to the general saponification and decarboxylation procedure; chiral HPLC: Chiralcel OJH: hex: $i \mathrm{PrOH}=95: 5, t_{\mathrm{R}}=7.9$ min. $(S), 8.5 \mathrm{~min}$. $(R)$.

3-(1,3-Benzodioxol-5-yl)-3-(3,4,5-trimethoxyphenyl)propanoic acid (11b). ${ }^{12}$ Obtained from 10b as a racemic solid according to the general saponification and decarboxylation procedure; ${ }^{1} \mathrm{H} \mathrm{NMR}\left(\mathrm{CDCl}_{3}\right) \delta$ 6.790-6.63 (comp, $\left.3 \mathrm{H}\right), 6.43(\mathrm{~s}$, $2 \mathrm{H}), 5.93(\mathrm{~s}, 2 \mathrm{H}) 4.39(\mathrm{t}, J=7.9 \mathrm{~Hz}, 1 \mathrm{H}), 3.81(\mathrm{~s}, 9 \mathrm{H}), 3.01(\mathrm{~d}, J=7.9 \mathrm{~Hz}, 2 \mathrm{H}) ;{ }^{13} \mathrm{C} \mathrm{NMR}\left(\mathrm{CDCl}_{3}\right) \delta 176.4,153.3,147.9$, $146.4,139.0,137.1,120.4,108.3,108.2,104.7,101.1,60.9,56.2,46.7,40.7$.

(3S)-3-(2-Methylphenyl)-3-phenylpropanoic acid (11c). Obtained as a waxy solid from 10c according to the general saponification and decarboxylation procedure; chiral SFC: chiralcel OJ column, $4 \% \mathrm{MeOH}$ in $\mathrm{CO}_{2}$ for 4 min. then ramp to $40 \% \mathrm{MeOH}$ at $2 \%$ per min, $1.5 \mathrm{~mL} / \mathrm{min} ., 35^{\circ} \mathrm{C}, 25 \mathrm{~min}$. run time, $t_{\mathrm{R}}=12.3 \mathrm{~min} .(R), 13.8 \mathrm{~min}(S) ;{ }^{1} \mathrm{H} \mathrm{NMR}\left(\mathrm{CDCl}_{3}\right) \delta 11.7$ (brs, $1 \mathrm{H}), 7.31-7.18$ (comp, $9 \mathrm{H}), 4.77(\mathrm{dd}, J=7.8,7.9 \mathrm{~Hz}, 1 \mathrm{H}), 3.12(\mathrm{dd}, J=7.8,16.1 \mathrm{~Hz}, 1 \mathrm{H}), 3.07$ (dd, $J=7.9,16.1 \mathrm{~Hz}$, $1 \mathrm{H}) ; 2.33(\mathrm{~s}, 3 \mathrm{H}) ;{ }^{13} \mathrm{C}$ NMR $\left(\mathrm{CDCl}_{3}\right) \delta 178.2,142.8,140.9,136.2,130.7,128.5,127.8,126.6,126.4,126.2,126.1,42.6$, 40.8, 19.7; IR (neat) $\delta 3023,2927,1611,1704,1491,1433,1310,1210,1154,753 \mathrm{~cm}^{-1}$; CHN calcd. for $\mathrm{C}_{16} \mathrm{H}_{16} \mathrm{O}_{2}$ [240.30]: C, 79.97; H, 6.71. Found: C, 79.94; H, 6.75 .

\footnotetext{
${ }^{6}$ Kanemasa, S.; Suenaga, H.; Onimura, K. J. Org. Chem. 1994, 59, 6949.

${ }^{7}$ Chiefari, J.; Galanopoulos, S.; Janowski, W. K.; Kerr, D. I. B.; Prager, R. H. Aust. J. Chem. 1987, 40, 1511-1518

${ }^{8}$ Maguire, A. R.; Buckley, N. R.; O'Leary, P.; Ferguson, G. J. Chem. Soc., Perkin Trans. 1 1998, 4077-4091.

${ }^{9}$ Enholm, E. J.; Cottone, J. S.; Allais, F. Org. Lett. 2001, 3, 145-147.

${ }^{10}$ Enholm, E. J.; Cottone, J. S. Org. Lett. 2001, 3, 3959-3962.

${ }^{11}$ Maguire, A. R.; O'Leary, P.; Harrington, F.; Lawrence, S. E.; Blake, A. J. J. Org. Chem. 2001, 66, 7166-7177.

${ }^{12}$ Pettit, G. R.; Baumann, M. P.; Rangammal, K. N.
} 